\title{
Creación de competencias \\ en ambientes locales y redes productivas
}

\section{Gabriel Yoguel \\ Investigador docente \\ Instituto de Industria Nacional, \\ Universidad Nacional \\ de General Sarmiento, \\ República Argentina \\ Yoguel@mail.retina.ar}

En el nuevo escenario internacional, caracterizado por nuevas tecnologías que hacen uso intensivo de información, por la globalización de los mercados y por el aumento de la presión competitiva y la incertidumbre que enfrentan los agentes, la competitividad es un fenómeno sistémico. La capacidad endógena de los agentes, el grado de desarrollo del ambiente en que actúan y la pertenencia a una red productiva se han convertido en elementos claves para desarrollar competencias y crear ventajas competitivas. Se comienza a afirmar que las ventajas competitivas de los países, regiones y agentes no se derivan necesariamente de su dotación factorial, sino también de factores intangibles que se construyen a partir del desarrollo de competencias endógenas y de la articulación con otros agentes. En el tránsito de las ventajas comparativas estáticas a las dinámicas, la capacidad de aprender, concebida como un proceso interactivo y socialmente embebido, desempeña un papel clave. En este artículo se analiza cuáles son y de qué dependen los mecanismos endógenos de creación de competencias y de transformación de conocimientos genéricos en específicos entre los agentes individuales, en las tramas productivas y en los diferentes ambientes locales. Se muestra la importancia que en los últimos años ha dado la teoría económica a la relación entre la tecnología y el desarrollo de procesos de aprendizaje, en especial a partir de los enfoques neoschumpeterianos y evolucionistas. Se efectúa una aproximación a la forma como los agentes económicos aprenden, transforman conocimiento genérico en específico y articulan los saberes codificados y tácitos. Y por último se pone de relieve que estos procesos no son consecuencia de un desarrollo natural y lineal de los sistemas productivos, sino de un largo proceso evolutivo de aprendizaje. 


\section{Introducción}

En los últimos veinte años se han registrado transformaciones importantes en el ámbito internacional, entre las que destacan la globalización de los mercados, la generalización de los procesos de apertura y la aparición de nuevos paradigmas técnicos y de organización que implican un uso intensivo de la información. Estas transformaciones han puesto en tela de juicio el concepto de la competitividad como un fenómeno de naturaleza exclusivamente macroeconómica y sectorial, determinado por ventajas comparadas estáticas o por la dotación factorial.

En efecto, la volatilidad de la demanda, la segmentación de los mercados, el acortamiento del ciclo de vida de los productos, las incertidumbres estratégicas asociadas con la nueva situación mundial y la posibilidad de combinar economías de escala y de variedad, han significado un aumento considerable de la presión competitiva que deben enfrentar los agentes económicos. A los tradicionales factores macroeconómicos y sectoriales que constituían antes los elementos claves de la competitividad, se suman ahora los elementos que dependen de la gestión de los agentes y de la naturaleza del medio económico y social en el que actúan. En consecuencia, la competitividad se plantea como un fenómeno sistémico y tanto la conducta de los agentes como el grado de desarrollo del medio local adquieren gran importancia en la creación de ventajas competitivas. Se comienza, así, a afirmar la convicción de que las ventajas competitivas de los países, regiones y agentes no derivan necesariamente de su dotación de factores.

Las nuevas conceptualizaciones parten de la base de que las ventajas comparativas se pueden crear y por lo tanto son de naturaleza dinámica. En el tránsito desde las ventajas comparativas estáticas a las dinámicas tienen un papel clave la tecnología y el desarrollo de los procesos de aprendizaje. La capacidad de aprender - concebida como un proceso interactivo incor-

$\square$ Una versión de este trabajo fue presentada en el Seminario de Sistemas Locales de Innovación realizado en Rio de Janeiro el 14 y 15 de diciembre de 1998. Agradezco los comentarios de mis compañeros del Instituto de Industria, de Graciela Gutman y de Bernardo Kosacoff a versiones previas de este trabajo y las observaciones de uno de los jueces anónimos que colaboran con la $R e$ vista de la CEPAL. porado en la trama social- y el desarrollo de "competencias en los agentes" determinan el éxito económico de las empresas, regiones y países (Ernst y Lundvall, 1997). Por lo tanto, en este nuevo contexto resultan de la mayor importancia para la creación de ventajas comparativas las reacciones de la empresa tendientes a idear, planificar y llevar adelante el desarrollo y la mejora de productos y procesos, a introducir cambios de organización y a establecer nuevas formas de vinculación con el mercado. Es decir, en el proceso de competencia y en la búsqueda de diferenciación los agentes apuntan a mejorar su capacidad innovativa — technological capabilities (Lall 1992)—, entendida como el potencial para transformar los conocimientos genéricos en conocimientos específicos a partir de competencias estáticas y dinámicas derivadas de un aprendizaje formal y oficioso (Boscherini y Yoguel, 1996a). Las competencias estáticas pueden definirse como el conjunto de conocimientos y habilidades tecnológicos y de organización - formales e informales-que los agentes generan para llevar a cabo los proyectos mencionados. No se reducen a información y equipos, sino que incluyen también la capacidad de organización, los patrones de conducta y las rutinas que influyen en el proceso de toma de decisiones.

En el presente artículo se pretende pasar revista a los últimos avances en esta materia de que dan cuenta las publicaciones más recientes, para comprender cuáles son y de qué dependen los mecanismos endógenos de creación de competencias y de transformación de conocimientos genéricos en específicos tanto en los agentes como en las redes productivas y en los distintos ambientes locales. Interesa conocer cómo los agentes económicos aprenden y transforman conocimiento genérico en específico, en resumidas cuentas, cómo innovan.

Los estudios que se examinan con tal propósito se originaron en los países desarrollados y se refieren especialmente a la nueva importancia de los procesos de aprendizaje en la creación de ventajas competitivas en los distritos industriales, en los sistemas de empresas (clusters) y en lo que se denomina la nueva competencia territorial (Poma, en prensa). Sin embargo, el análisis es también relevante para América Latina. A pesar de que en los años noventa se consolidaron en 
la región las tendencias hacia una creciente primarización de la estructura productiva y del perfil de especialización comercial (CEPAL, 1996), es evidente que la disminución de las diferencias de productividad con respecto a los países más desarrollados, la sustentabilidad en el tiempo de los modelos que se están aplicando y la disminución de las desigualdades en la distribución del ingreso exigirán un mayor nivel de complejidad y la incorporación de una mayor proporción de conocimientos codificados y tácitos en las funciones de producción de los agentes. Cabe señalar que en algunas agroindustrias que se han abierto camino en este nuevo sistema de especialización industrial, el proceso de producción ha alcanzado en los últimos años un grado de complejidad cada vez mayor, lo que se manifiesta en la creciente importancia de las tecnologías que hacen uso intensivo de información (Gutman, 1999).

En la Sección II de este artículo se analiza el papel de los procesos de aprendizaje en la teoría económica, destacando los nuevos enfoques teóricos. Luego, en la Sección III, se examina el papel del conocimiento tácito y el desarrollo de las ventajas competitivas en las competencias técnica y laboral de los agentes y en la Sección IV se analizan los procesos de aprendizaje en el ámbito local y en los sistemas de empresa. Finalmente, en la Sección V se presentan algunas conclusiones.

\section{II}

\section{La tecnología, la firma y la generación de conocimiento: los nuevos abordajes teóricos}

En los últimos quince años la vinculación entre la tecnología y el desarrollo de procesos de aprendizaje ha cobrado renovado impulso en la teoría económica y el tema se halla en continua evolución. En ese contexto, es endeble la correspondencia entre el marco teórico de la tecnología en uso corriente y la aparición de estos nuevos procesos en la sociedad. Por ejemplo, la teoría económica neoclásica estándar parte de una concepción de la empresa según la cual los agentes económicos son sujetos maximizadores, que operan en condiciones de racionalidad e información perfectas, y que se mueven en un medio sin incertidumbre o con expectativas racionales. En ese marco, el capital se supone maleable — moldes de jalea — y la tecnología se concibe como un conjunto de técnicas productivas —una biblioteca que contiene todo el conocimiento posible al alcance de los agentes - que pueden ser escogidas y captadas sin dificultad a costo cero en función de la relación entre la tasa de utilidad y la escala de salarios (Solow, 1963). Para elegir las técnicas óptimas, los agentes sopesan los últimos adelantos en ese conjunto y la relación salario-beneficios vigente. Al pasar de relaciones beneficios/salario elevadas a otras más bajas, los agentes cambian las técnicas con uso intensivo de trabajo por otras con uso intensivo de capital. Se supone además que existe un ordenamiento de las técnicas funcional a los rendimientos decrecientes de los factores y que los umbrales de precio de los factores correspondientes a cada técnica elegida se traspasan sólo una vez en el sentido previsto por una función de producción con rendimientos decrecientes (Samuelson, 1962). En consecuencia, una técnica que haga uso intensivo de trabajo con un nivel elevado de utilidad no puede ser empleada a una tasa menor y se excluye - por lo tanto- la posibilidad de recambio de técnicas. La elección de técnicas y su reemplazo ante cambios en las condiciones de distribución se efectúa en forma instantánea y no exige un proceso de aprendizaje.

En este marco neoclásico estándar, que sobrevivió al antiguo debate sobre la teoría del capital a pesar del cambio de posición de sus participantes, ${ }^{1}$ el

\footnotetext{
${ }^{1}$ Samuelson (1962) admite que no puede ser universalmente válido el cuento sencillo de Jevons, Bohm-Bawerk, Wicksell y otros autores neoclásicos, según el cual a medida que baja la tasa de interés como consecuencia de la abstención del consumo presente a favor del consumo futuro, la tecnología debe volverse en algún sentido más indirecta, más mecanizada y más productiva. Al final del trabajo, reconoce que las críticas de Pasinetti, Morishima, Garegnani y otros merecen nuestra gratitud por haber demostrado que la reversión es una posibilidad lógica en cualquier tecnología. En una demostración de honestidad intelectual poco frecuente, Samuelson plantea que si todo esto causa dolores de cabeza a quienes suspiran por las viejas parábolas de la teoría neoclásica, deberemos recordarles que los académicos no han nacido para llevar una existencia fácil. Debemos respetar y evaluar los hechos de la vida.
} 
progreso técnico se supone no incorporado en la función de producción e independiente de la acumulación de capital. Por lo tanto, la adquisición de conocimientos y el proceso de aprendizaje de los agentes no constituye un argumento explicativo ligado a la función de producción y se considera independiente del capital social de los agentes. Aunque algunos modelos incorporan la existencia de curvas de aprendizaje a las que se adjudica el desplazamiento de la función de producción - progreso técnico no incorporado-, este aprendizaje es exógeno e independiente de los factores de producción (en especial del capital social) y por lo tanto no influye en ellos.

La falta de aplicación empírica de esta teoría para explicar los cambios producidos en la estructura productiva generó otras interpretaciones que -a la luz de la nueva teoría del comercio internacional y de la teoría del crecimiento- hicieron modificar algunos supuestos del modelo neoclásico estándar. Así, por ejemplo, Grossman y Helpmann (1992) desarrollan una teoría del crecimiento que endogeneiza el progreso técnico en la función de producción y que muestra que la posibilidad de interacción estratégica entre agentes, las actividades de investigación y desarrollo, la existencia de economías de escala y los procesos de aprendizaje resultan centrales para la creación de ventajas competitivas y para explicar el patrón de especialización de los agentes en el comercio exterior. A partir de un esquema de equilibrio general, consideran que las decisiones de los agentes de invertir en investigación y desarrollo ${ }^{2}$ y los beneficios de las innovaciones asociadas a ellos dependen de las condiciones de funcionamiento del conjunto del mercado. Así, el proceso de acumulación de conocimientos genera en forma endógena los incrementos de la productividad que sostienen el crecimiento.

Sin embargo, el cambio más importante en lo que se refiere a la incorporación explícita del conocimiento en la teoría deriva de las diversas corrientes neoschumpeterianas y evolucionistas que se apartaron de la teoría del equilibrio general e implícitamente aceptaron la existencia de transacciones en condiciones de desequilibrio. ${ }^{3}$ En forma muy estilizada, estas teorías

\footnotetext{
${ }^{2}$ En ese marco teórico, el proceso de generación de conocimientos se limita a los laboratorios de investigación y desarrollo formales. 3 Aunque no se trata aquí de enmarcar el trabajo en alguna concepción epistemológica particular, interesa señalar que los distintos elementos del cuerpo teórico neoschumpeteriano — que rompe con la lógica de análisis neoclásico y que en algunos casos retoma el análisis clásico - podrían converger hacia un programa de investigación, en el sentido planteado por Lakatos (1983). Así, se avanza
}

parten de una concepción distinta de la empresa y de la tecnología y asignan un papel clave a los procesos de aprendizaje tanto formales como informales que aplican los agentes en la generación de ventajas competitivas. La teoría de la empresa considera entre sus argumentos la racionalidad acotada de los agentes, el acceso imperfecto a la información y la incertidumbre no modelable del medio en que actúan. La incertidumbre - que constituye un elemento esencial del análisis- es un parámetro que los agentes no pueden expresar en términos probabilísticos: la información incompleta no puede ser completada y los agentes toman sus decisiones con ese lastre. En consecuencia, el potencial de los agentes para transformar conocimientos genéricos en específicos -el desarrollo de su capacidad innovadora (Lall, 1992; Boscherini y Yoguel, 1996a) — influye decisivamente en las posibilidades de generar ventajas competitivas y disminuir en parte las incertidumbres estratégicas existentes en los mercados en los que concurren. ${ }^{4}$ En esas teorías se parte de la idea de que una condición necesaria para generar estos procesos de aprendizaje es disponer de un umbral mínimo de "competencias" (Rullani, en prensa), que se hace menos significativo en los "medios" y países en que se generan externalidades positivas y el sistema nacional de innovación funciona en forma adecuada.

Por su parte, como señalan Dal Bó y Kosacoff (1998), el conocimiento no puede ser expresado com-

hacia un núcleo firme compuesto por i) una teoría de la empresa que supone que los agentes tienen racionalidad acotada, imperfecta información e incertidumbre y que tienen que tomar decisiones en ese contexto; ii) una teoría sobre la tecnología y el cambio técnico que asigna un papel clave al proceso de innovación entendido como el proceso de transformación de conocimiento genérico en específico y de asimilación de conocimiento codificado y tácito. A su vez, la teoría predice hechos nuevos todavía difíciles de captar empíricamente entre los que destaca que el conocimiento experimental y tácito resulta decisivo en el desarrollo de la capacidad de competencia de los agentes. En este marco teórico, las interrelaciones entre el conocimiento no probado y experimental (doxa) y el conocimiento probado (episteme) están excluidas en la mayor parte de la epistemología anterior y posterior a Popper.

${ }^{4}$ Según Poma (1998), el nuevo escenario internacional viene acompañado de un menor grado de control de las incertidumbres por parte de los agentes. Así, por ejemplo, en el marco del fordismo la incertidumbre se podía controlar tanto desde el lado de la oferta (con la creación de fases de producción en línea, automáticas y repetitivas) como desde el lado de la demanda (con la creación de nuevas necesidades en los consumidores). En el caso de los distritos industriales, la incertidumbre existente en las condiciones del mercado se enfrentaba a través de la certidumbre de los valores compartidos y del sistema de relaciones personales, y las incertidumbres tecnológicas a partir de innovaciones incrementales. Por el contrario, afirma, en el nuevo escenario ha aumentado la incertidumbre porque ha aumentado la complejidad competitiva. 
pletamente en forma explícita y, en consecuencia, no se puede transformar ni convertir en información como bien transable. Esta característica del conocimiento introduce fuertes especificidades en la concepción de la tecnología, que se considera no sólo como un acervo de máquinas y técnicas de producción ${ }^{5}$ sino fundamentalmente como un sistema complejo de generación y difusión de conocimiento codificado y tácito (Bell y Pavitt, 1995) acumulado por la empresa (Ernst y Lundvall, 1997).

Esta concepción de la tecnología comprende dos aspectos no incluidos en el análisis neoclásico (Metcalfe, 1998). Por un lado se incorpora el análisis de la capacidad de los individuos y la competencia generada al interior de una organización, entendidas como el conjunto de conocimientos, rutinas, procedimientos, habilidades y prácticas del que puede disponer. Esta competencia es algo más que el conjunto de conocimientos técnico-ingenieriles pues incluye cuestiones de organización y gestión vinculadas con la marcha del proceso productivo. (David, 1985). Con esta concepción, el progreso técnico es un conjunto sucesivo de innovaciones incrementales —mucho más significativas que en el periodo anterior - que complementan las innovaciones radicales y que en algunos sectores son las más significativas. Estas mejoras permiten producir con un volumen determinado de recursos, una mayor cantidad de producto, de calidad superior o en forma más eficiente. Además, la capacidad de pensar y el desarrollo de procesos de aprendizaje aplicados forman parte de la tecnología. La literatura que trata el tema de las trayectorias y paradigmas tecnológicos (Pérez, 1985) considera que el progreso técnico logrado por la empresa toma como punto de partida los equipos, insumos y capacidades incorporados en los individuos y las organizaciones. Estos elementos, sumados al uso de lo que se denomina el "sentido común técnico" (Pérez, 1983), les permite a los agentes introducir ya sea innovaciones incrementales en la tecnología vigente o cambios radicales en aquellas ramas que no hayan alcanzado aún el paradigma tecnológico ideal.

Así, las empresas no eligen la técnica óptima en la "biblioteca de conocimiento técnico", sino que, por

\footnotetext{
${ }^{5}$ Este nivel de análisis se asocia con la visión neoclásica según la cual la tecnología es el conjunto de bienes de capital y procesos de producción incorporados en la maquinaria o enteramente transferibles a través de manuales. Desde ese punto de vista, el cambio tecnológico es el proceso por el cual las economías cambian con el tiempo con respecto a los productos que producen y los procesos usados para fabricarlos.
}

el contrario, deben hacer esfuerzos de selección y adaptación que requieren como condición necesaria umbrales mínimos de conocimientos codificados y en especial tácitos. Mientras el componente codificado ${ }^{6} \mathrm{del}$ proceso de conocimiento es básicamente transable, el componente tácito ${ }^{7}$ es específico de la empresa, no se puede comprar en el mercado y constituye un punto clave en las diferencias tecnológicas y en las ventajas competitivas específicas de los agentes (Lall, 1995). Según Poma (en prensa), dado que el conocimiento tácito podría ser definido como la sedimentación del conjunto de memorias que incluyen las secuencias de operaciones que permiten alcanzar con éxito distintos objetivos, el uso de un lenguaje común (formal o informal) resulta necesario para que el conocimiento pueda circular y difundirse. En ese sentido, "una parte del conocimiento tácito tiene que colocarse en el marco de algunos parámetros formales de pensamiento". Por lo tanto, el lenguaje puede ser interpretado como una "institución" de carácter tanto formal como informal que puede facilitar o limitar el desarrollo del proceso de aprendizaje (Poma, en prensa).

Cuando el acceso a los conocimientos codificados se generaliza para todos los agentes, éste no se convierte en un elemento diferenciador de conductas y rendimientos. En cambio, cuando no todos los agentes pueden acceder a los conocimientos codificados por tener información imperfecta, por no disponer de la competencia mínima necesaria o por no reunir los conocimientos tácitos mínimos para asimilarlos, entonces la desigualdad de acceso a los conocimientos codificados puede también constituir un elemento diferenciador de conductas.

Según Lall (1995), para la creación de ventajas competitivas no sólo es necesario manejar la tecnología en un sentido estático - llegar a los niveles fija-

\footnotetext{
${ }^{6}$ Los conocimientos codificados incluyen el conjunto de conocimientos transmisibles de tipo tecnológico (incorporados en materiales, máquinas, componentes y productos finales), organizacionales y transmisibles por interacción comunicativa (Internet, cursos, etc.) a los que se accede a través del mercado (Becattini y Rullani, 1993). ${ }^{7}$ El conocimiento tácito supone: i) el conocimiento no codificado en manuales sobre la tecnología aplicada al proceso de trabajo, ii) el conocimiento general y conductual, iii) la capacidad de resolución de problemas no codificados, y iv) la capacidad para vincular situaciones e interactuar con otros recursos humanos. En suma, el conocimiento tácito permite efectuar una representación mental compleja del proceso de trabajo (Novick, 1998). Este tipo de atributos que se exige a los trabajadores (que no pueden ser expresados o bien no pueden ser formalizados completamente) están fuertemente influidos por el contexto (Mertens, 1996), y se adquieren en diversas situaciones como lugares de trabajo, asociaciones, intercambios informales (Ducatel, 1998).
} 
dos por los manuales - sino desarrollar procesos de aprendizaje (con curvas no predecibles) que les permita a las empresas lograr mejores productos y procesos, introducir cambios en la organización y aumentar la complejidad de los encadenamientos con el sistema local. Según Ernst y Lundvall (1997), incluso para aplicar en la práctica el conocimiento codificado (interpretación de manuales de ingeniería y diseño, aprovechamiento de conocimientos científicos y de gestión de tipo genérico, especificación de los criterios de calidad, etc.), la organización necesita también conocimientos tácitos que se manifiestan en las rutinas de organización y en la experiencia colectiva de grupos específicos de la empresa en investigación y desarrollo, gestión, producción y comercialización. En consecuencia, el desarrollo de competencias tácitas al interior de la empresa representa un activo intangible difícil de transferir, que puede tener un efecto positivo sobre los resultados de la gestión y transformarse en una barrera que impida la entrada al mercado a los agentes que no tienen ese tipo de conocimientos. ${ }^{8}$

Según Nightingale (1996), como el desarrollo del conocimiento - inserto en la trama social - depende de la capacidad innata de los individuos para reconocer similitudes, los elementos tácitos tiene un papel decisivo. ${ }^{9}$ Aprender no es acumular más información, sino reconocer comportamientos y conexiones entre los elementos que están en la memoria, lo que depende de los conocimientos acumulados por la experiencia y de la capacidad automática que tienen los individuos de vincular la experiencia con el conocimiento.

En ese marco, algunos autores sugieren que el grado de relación recíproca entre los conocimientos codi-

\footnotetext{
${ }^{8}$ Como casi todos los indicadores de la intensidad del conocimiento se refieren a la educación formal y a los esfuerzos de investigación y desarrollo, se distorsiona la imagen de la economía del aprendizaje, que no refleja la importancia de los procesos incrementales de innovación en toda la organización. Para captar el desarrollo del conocimiento codificado y tácito, no centrado en unidades específicas de investigación y desarrollo, es preciso partir de otros indicadores de tipo cualitativo y cuantitativo y de la idea de que la capacidad creadora está difundida en toda la organización (Lassini, 1992). El progreso en esa dirección en Argentina se advierte en Boscherini y Yoguel (1996b), Boscherini, López y Yoguel (1997), Rearte, Lanari y Alegre (1997) y Moori-Koenig y Yoguel (1998). ${ }^{9}$ Como señala Nightingale (1996), el conocimiento tácito es fundamental para entender el significado de la palabra cortar en expresiones como i) cortar una torta y ii) cortar el pasto. Aunque se usa la misma palabra en ambos casos, lo que se asocia con cortar varía en cada contexto. La palabra cortar tiene así un significado distinto que depende del conocimiento experimental acumulado previamente (tácito), que a la vez difiere entre distintos sujetos. Es decir, el significado de cortar está asociado con el conocimiento tácito previo, connotación que no da la palabra aislada.
}

ficados y los tácitos influye decisivamente sobre la eficiencia alcanzada en el proceso de aprendizaje de la empresa. Estos procesos de aprendizaje, que se generan en sectores con desigual grado de desarrollo tecnológico, suponen no sólo actividades formales de capacitación y de investigación y desarrollo sino también un conjunto de actividades informales, que los agentes no siempre reconocen como tales (aprender haciendo, aprender por interacción, aprender produciendo). Estos procesos de aprendizaje de distinto tipo se van acumulando durante la vida activa de los agentes y se concretan en activos tangibles e intangibles de vital importancia para la competencia. Sin embargo, estos activos no son eternos, porque deben ser evaluados en el proceso de desarrollo de competencias. Mientras algunos se degradan y se devalúan por no alcanzar los umbrales mínimos requeridos en el mercado, otros resultan ganadores y van configurando los elementos del patrón tecnológico predominante.

Para precisar los distintos tipos de aprendizaje y su influencia en la competitividad de los agentes, Johnson y Lundvall (1994) desarrollaron una tipología en que distinguen cuatro tipos de conocimientos, vinculados con su carácter tácito o codificado. El conocimiento que denominan know what (saber qué), se asimila a lo que normalmente se clasifica como hechos, o información, mientras que el conocimiento denominado know why (saber por qué) es de carácter científico y se refiere a los principios y leyes de movimiento de la naturaleza. Ambos son en esencia de carácter codificado y pueden ser adquiridos en el mercado en la forma de libros, cursos, bases de datos y fuentes similares. En cambio, los conocimientos que se denominan know-how (saber cómo) y know-who (saber quién) son de tipo tácito. El primero se refiere a las destrezas que se adquieren a partir de la experiencia directa en actividades productivas y de gestión. El segundo se vincula con los conocimientos desarrollados y mantenidos dentro de una empresa o en grupos de investigación. Las empresas pueden tener acceso a este tipo de conocimiento no sólo a partir de actividades propias sino a través de la cooperación empresarial y las alianzas estratégicas. En especial el acceso al know-who exige contacto y comunicación directa entre individuos y el desarrollo de relaciones de confianza. En estas condiciones y como se aprende en la práctica social, los agentes de mayor desarrollo relativo (por ejemplo los del Silicon Valley) se vinculan con redes formales e informales de científicos que llevan a cabo diversos programas de investigación básica susceptibles de aplicación específica. Siendo así, este tipo de conoci- 
miento no se suele transferir por los canales formales de información. ${ }^{10}$

Como sugiere Ducatel (1998), las cuatro formas de conocimiento tienen fuertes lazos de interacción. El desarrollo del conocimiento codificado (know-what $\mathrm{y}$ know-why) y la réplica de resultados experimentales dependen del conocimiento tácito y del reconocimiento del know-who en el campo científico y tecnológico. Dada la racionalidad acotada de los agentes, en condiciones de incertidumbre (respecto al futuro del mercado o la homogeneidad de los insumos, por ejemplo), con frecuencia la condición necesaria para el desarrollo e incorporación de conocimientos codificados es la existencia de elementos tácitos previos que no son fáciles de codificar. Por otro lado, el conocimiento tácito, que tiene su origen en la complejidad y en las variaciones de calidad y que se impone en situaciones de incertidumbre, en las que es necesario usar simultáneamente diferentes aptitudes humanas y relacionar diversos parámetros, también exige un mínimo de conocimiento codificado previo. La globalización y las tecnologías de la información, al aumentar las incertidumbres estratégicas de los agentes, si bien abaratan y facilitan el acceso a opciones codificadas vía Internet, refuerzan las razones para desarrollar el conocimiento tácito.

La importancia que adquieren los elementos tácitos del conocimiento en el nuevo contexto para el éxito a largo plazo de los agentes, que se manifiesta en la capacidad de adaptarse al cambio (flexibilidad) y en la capacidad de imponer cambios (innovación), contrasta con la falta de esos elementos en la función de producción que representa la mayoría tradicional. ${ }^{11}$

Así, en un marco analítico de información y racionalidad imperfectas, la empresa enfrenta un escenario con incertidumbre que no puede prever y en el que la tecnología no se reduce a la compra de máquinas acompañadas de información codificada. Los factores cognoscitivos y el desarrollo de procesos de aprendizaje formales e informales asumen una importancia vital para el desarrollo de la capacidad de innovación y - por lo tanto- para la capacidad de competencia de los agentes.

Este papel creciente de los procesos cognoscitivos en la construcción de activos tangibles e intangibles se da entonces no sólo al interior de las organizaciones —organizaciones inteligentes (Bessant, 1991)— sino también en los medios en que actúan las empresas (regiones inteligentes). Al interior de las organizaciones comienza a desarrollarse la idea de empresa calificante y a manifestarse una transición de la demanda de calificaciones típicas de la organización fordista a la demanda de competencias, que se manifiesta como una nueva tendencia que coexiste aún con modalidades anteriores. $^{12}$

\section{III}

\section{Las competencias técnicas y laborales de los agentes: el conocimiento tácito y el desarrollo de ventajas competitivas}

En el desarrollo de conocimientos técnicos en la empresa y en la posibilidad de que ésta se apropie de conocimiento codificado y tácito - en un contexto caracterizado por la racionalidad acotada de los agentes, la información imperfecta, la incertidumbre sobre la situación del mercado y un cambio técnico aceleradoinfluye en alto grado el perfil particular de competencia laboral de los recursos humanos. Esta competen-

\footnotetext{
${ }^{10}$ Este tipo de transferencia de conocimientos se efectúa a partir de diversos mecanismos de vinculación entre centros universitarios y empresas.
}

\footnotetext{
11 La creciente importancia de los aspectos tácitos del proceso de aprendizaje ha restado importancia a los métodos tradicionales de medición centrados en variables vicarias de los aspectos formales del aprendizaje de las organizaciones (laboratorios de investigación y desarrollo, patentes, etc.). Al respecto véase, entre otros, Malerba (1993), Acs y Audrescht (1988), Lassini (1992), Malerba y Orsenigo (1993) y Boscherini y Yoguel (1996b).

12 Los autores neoschumpeterianos comentados en esta sección consideran que en el desarrollo de procesos de aprendizaje, los agentes económicos combinan conocimiento "científico" codificado con otro tipo de conocimiento que puede incluir elementos tanto racionales como irracionales con fuerte contenido inductivo. Se alejan por lo tanto, desde el punto de vista epistemológico, del falsacionismo popperiano. Es decir, a lo largo de su proceso de aprendizaje, los agentes evolucionan en el sentido planteado por Lamarck (pueden corregir sus trayectorias) más que por un proceso de selección natural darwiniano (Gómez, 1995).
} 
cia laboral, considerada como el conjunto de conocimientos de diverso origen y naturaleza que tienen los trabajadores de una organización, presenta una serie de características que deben ser validadas en el mercado y que operan en situación de incertidumbre (Gallart, 1998). Estas características se refieren a la capacidad de resolver problemas, de aprender y de difundir conocimientos a la organización a partir de ciertas calificaciones básicas (Cariola y Quiroz, 1998), de gestionar recursos e información, de desarrollar relaciones interpersonales, de tener dominio sobre la tecnología (Mertens 1996) ${ }^{13}$ y de analizar y seleccionar opciones entre un conjunto de alternativas (Novick, Bartolomé, Buceta, Millavares y Senén González, 1998). A diferencia de las calificaciones tradicionales de los recursos humanos que podían validarse con un certificado de formación, la competencia sólo puede ser validada en situaciones laborales específicas (Novick, Bartolomé, Buceta, Millavares y Senén González, 1998).

Como señala Mertens (1996), se exige del trabajador un complejo conjunto de atributos, entre los que destacan la capacidad para asumir mayor responsabilidad, para comunicarse, para resolver problemas y para aprender, así como el disponer de destrezas mentales y manuales. En ese conjunto, la capacidad de aprender es elemento primordial por la mayor complejidad de los sistemas de innovación. Ducatel (1998) sostiene que las competencias requeridas en el proceso de trabajo incluyen i) capacidad para manejar modelos mentalmente; ii) comprensión del funcionamiento y de la interacción de las máquinas; iii) posibilidad de efectuar deducción estadística; iv) capacidad de comunicación oral y visual; v) responsabilidad individual por el proceso de trabajo y el producto; vi) buen criterio, y vii) habilidad para combinar asuntos técnicos y de negocios. En lo que el autor citado denomina el triángulo del proceso de aprendizaje interactúan el conocimiento teórico, el vocacional y el experimental, lo cual exige un fuerte vínculo entre el proceso de trabajo y el sistema educativo, que raras veces se da. En un medio en que el trabajo es cada vez menos observable y en gran parte ocurre en la cabeza del trabajador (Hanser, 1995), la destreza laboral supone nuevos conocimientos básicos y técnicos, conductuales e intelectuales (Novick, Bartolomé, Buceta, Millavalles y Senén González, 1998). ${ }^{14}$

En el proceso de desarrollo de competencias, los agentes pueden apropiarse de conocimientos tácitos o generales, aplicando mecanismos de cooperación que contribuyan a su circulación o creando una organización particular del proceso de trabajo que permita y estimule la circulación, apropiación y generación de conocimiento tácito. La capacidad de las empresas para hacer frente a las presiones competitivas depende de la combinación de los conocimientos codificados y tácitos que elaboran en su interior. De ahí que su potencial para llevar adelante ese proceso depende de la competencia inicial y de su trayectoria previa, de los grados de libertad de los patrones tecnológicos, del medio local, de su inclusión en redes en las que ocurran estos procesos y de la forma como organizan el proceso de trabajo y la producción.

Sin embargo, el aprendizaje de la empresa a base de conocimientos codificados y tácitos puede dar resultados positivos o negativos, originándose un proceso de destrucción creativa en el que algunas empresas generan soluciones que les permiten mantenerse en el mercado, otras desaparecen y otras surgen. En un contexto de multiplicidad de respuestas y de grados de libertad (Nelson, 1991) el mercado selecciona conductas de manera imperfecta, y por lo tanto no siempre sobreviven las mejores. ${ }^{15}$

La generación y circulación de conocimientos al interior de la empresa es un proceso complejo cuya intensidad depende de la necesidad de resolver problemas concretos en situación de incertidumbre, lo que estimula la demanda de soluciones no codificables; del grado de complejidad técnica de los equipos; del tipo de competencia básica de los agentes; de la capacidad de relacionarse y de trabajar en grupo y del grado de aprovechamiento de los conocimientos técnicos y de organización de los trabajadores.

\footnotetext{
${ }^{13}$ La gestión de información es la capacidad de los recursos humanos para buscar, evaluar, procesar, interpretar y comunicar información; la comprensión sistémica es la capacidad de comprender interpelaciones complejas y entender y diseñar sistemas; el dominio tecnológico se manifiesta en la capacidad para seleccionar y adaptar tecnologías y las relaciones interpersonales es la capacidad para interactuar con recursos humanos dentro y fuera de la organización, trabajar en equipo, enseñar y aprender.
}

\footnotetext{
${ }^{14}$ En América Latina se dan dos tendencias en la dirección contraria a la señalada. Por un lado, la primarización de la estructura productiva se asocia con una menor demanda de recursos humanos calificados. Por otro, la flexibilidad externa del mercado de trabajo conspira contra el desarrollo de la competencia en el sentido descrito.

${ }^{15}$ Las fallas en la selección de conductas constituyen un elemento clave que se toma en cuenta en las políticas encaminadas a crear mecanismos que las minimicen.
} 
En particular, la generación y la difusión del conocimiento tácito estarían asociadas con las características del capital humano de la empresa, la forma como se organiza el proceso de trabajo, ${ }^{16}$ y el grado de importancia que tiene para la empresa la interpretación y adaptación del conocimiento codificado externo. ${ }^{17}$ Para la difusión del conocimiento tácito hacia el interior de la empresa, el factor fundamental es la existencia de redes y distinto tipo de vinculación entre los agentes. El desarrollo del conocimiento tácito dentro de la empresa tiene un carácter sinérgico que, en buena medida, hace que los conocimientos de los individuos que forman parte de la organización sólo valgan en su interior y puedan devaluarse fuera de la empresa. Es decir, los conocimientos tácitos individuales se articulan entre sí para formar las ventajas competitivas de la organización y pierden parte de sus atributos fuera de ella.

Hasta que el conocimiento tácito se difunda y se logre codificar la pertenencia, los elementos tácitos en las empresas constituyen parte de su competencia, y se convierten en una ventaja competitiva. Para el agente $h$ la ventaja competitiva depende de la posesión de los conocimientos tácitos $1,2 \ldots . . m$. y para el agente $g$ la ventaja competitiva dependerá de los conocimientos tácitos $n \ldots . . . n+j$.

Dados dos agentes $h$ y $j$, sus ventajas competitivas pueden expresarse de la siguiente forma:

Ventaja competitiva $h=F(t 1, t 2, t 3, \ldots . t m)$

Ventaja competitiva $j=G(t n \ldots \ldots . . t n+j)$

La ventaja competitiva de $h$ es mayor que la de $j$ si los elementos tácitos $(1,2,3 . \ldots . . m)$ que tiene el agente $h$ tardan más tiempo en codificarse que los elementos $n, n+1, \ldots \ldots . n+j$ que tiene el agente $i$. En ese caso decimos que el agente $h$ se puede apropiar de ventajas competitivas durante un tiempo mayor. Cabe señalar

\footnotetext{
16 Grado de flexibilidad, tipo de jerarquía, existencia de células, grado de vinculación de los salarios individuales con el rendimiento del grupo al que pertenece.

17 Apunta a evaluar cuál es el desarrollo adicional que hace la empresa a partir de los bienes y servicios que compra u obtiene codificados y de los recursos humanos que contrata que la convierten en algo distinto, particular, específico no apropiable por otro agente (es decir, cambios en la disposición de las instalaciones, adaptación de tecnologías blandas, adaptación de información para el desarrollo de productos y procesos).
}

que el conocimiento puede identificarse como un factor de producción con algunas peculiaridades que lo alejan claramente del análisis neoclásico. Por una parte, la generación de conocimiento (su producción) aumenta con su consumo, lo que lo diferencia claramente de los factores de producción convencionales. Por la otra, las sinergias generadas a partir de la generación y difusión de conocimiento pueden implicar la existencia de rendimientos crecientes en los casos de vinculaciones virtuosas entre agentes públicos y privados.

Según Ducatel (1998), la importancia adquirida por el conocimiento tácito muestra las deficiencias del sistema educativo convencional para el desarrollo de la competencia de los agentes. La educación formal debe complementarse con la experiencia para que los agentes adquieran know-how. En consecuencia, aumenta la importancia de los aspectos interpersonales de las habilidades, el costado know how del proceso de conocimiento. Mertens (1996) efectúa un análisis pormenorizado de los elementos que deberían estar presentes en el sistema educativo formal para que sea funcional a la creación de las aptitudes requeridas en el sistema productivo. Sostiene que en el mundo actual "no se necesita una memorización sin sentido de asignaturas paralelas ni la adquisición de habilidades relativamente mecánicas sino conocimientos transversales que pueden ser actualizados en la vida cotidiana que se manifiestan en la capacidad de resolver problemas diferentes a los planteados en el aula escolar". ${ }^{18}$ Las habilidades constituyen un concepto multidimensional, que se basa en la capacidad física y cognoscitiva y de relaciones interpersonales que, si bien en general no son proporcionadas por el sistema educativo formal, requieren de éste como condición necesaria para su adecuado desarrollo. En el supuesto de que el proceso de conocimiento es un proceso social, para asegurar la competitividad son decisivas las relaciones que se desarrollan tanto al interior de la empresa como entre ésta y el resto de los agentes.

\footnotetext{
${ }^{18}$ Esto constituye un desafío importante para el sistema educativo, que funciona más bien como un ente encargado de la certificación de calificaciones que como un generador de competencias.
} 


\section{IV}

\section{La manifestación del proceso de aprendizaje: sistemas locales y redes de empresas}

De lo dicho se desprende que el aprendizaje en una organización es algo más que el desarrollo de procesos de entrenamiento y capacitación de tipo convencional. Nonaka y Takeuchi (1995) sostienen que la organización que aprende es aquella en la cual inventar nuevos conocimientos no es una actividad especializada, dominio de un grupo reducido, sino una forma de comportamiento general en que cualquiera es un trabajador con conocimiento. Lo que requiere una organización que aprende es una amplia variedad de sistemas y procedimientos para captar y movilizar el know-how, know-who, know-what y know-why de sus componentes, de modo de facilitar las diversas formas de conversión del proceso de conocimiento. ${ }^{19} \mathrm{El}$ objetivo de esos sistemas es involucrar a los trabajadores en las prácticas de aprendizaje y en la articulación y aplicación del conocimiento. Para lograrlo son necesarios la organización del trabajo en equipo, la existencia de procesos de capacitación formales e informales hechos a medida e incluidos en una perspectiva de largo plazo y el desarrollo de estrategias prácticas orientadas a que los trabajadores adquieran nuevas habilidades para que puedan llevar a cabo nuevas tareas y participar en rotaciones planificadas de los puestos.

Para Ducatel (1998) las organizaciones que aprenden exhiben un conjunto de características. En primer lugar i) tienen la capacidad de solución sistemática de problemas; suelen partir de un plan previo que se reexamina continuamente para consolidar experiencias y aplican un tipo de gestión que amplía la masa críti-

\footnotetext{
19 Aspecto fundamental del trabajo de estos investigadores es la definición de la dialéctica de los cuatro procesos de conversión del proceso de conocimiento y la forma organizacional que la posibilita: i) la conversión del conocimiento tácito en otro conocimiento tácito, fase denominada de "socialización del conocimiento"; ii) la conversión del conocimiento tácito en codificado, o fase de "externalización del conocimiento"; iii) la fase de combinación del conocimiento codificado, y iv) la conversión del conocimiento codificado en conocimiento tácito, denominada fase de "internalización del conocimiento". Junto con el desarrollo de estos procesos (socialización, externalización, combinación e internalización) plantean una forma de organización que se aparta tanto de los sistemas jerárquicos de arriba hacia abajo que se basan sólo en el predominio de conocimientos codificados, como de su opuesto, de abajo hacia arriba, que se sustenta sólo en la importancia asignada a los
} conocimientos tácitos. ca de ideas en la empresa, fomentando el cuestionamiento de las decisiones, y alienta el uso de técnicas estadísticas y el desarrollo del pensamiento interno guiado por la exactitud, la disciplina y la precisión; ii) tienen capacidad de experimentar con nuevos métodos, para lo cual fomentan la experimentación sistemática que ponga a prueba los nuevos conocimientos en los programas y proyectos en marcha, y premian la asunción del riesgo; se asegura así que las lecciones se transfieran efectivamente a toda la organización y se aprenda a transformar el conocimiento tácito (know how) en un know why codificado y transferible al interior de la organización y a otros agentes; iii) tienen la capacidad de aprender con la experiencia, lo que se concreta en el estudio de los errores pasados y en el registro sistemático de los resultados para que sean dados a conocer a todos los miembros de la organización, y iv) tienen la capacidad de aprender de los demás. Para ello, están abiertos, escuchan atentamente, analizan sistemáticamente los resultados, efectúan un análisis continuo de las mejores prácticas, planifican visitas y entrevistas, sacan ideas de los clientes sobre productos, competidores y cambios de preferencias, observan a los clientes en acción y pueden efectuar una transferencia de conocimiento rápida y eficiente preparando informes escritos, gráficos y orales. Este conjunto de características se da en forma muy específica en el proceso de aprendizaje de agentes que están al interior de sistemas locales o que forman parte de sistemas de empresas.

\section{La interacción entre lo local y lo global: el pa- pel del ambiente en el proceso de aprendizaje}

Por el carácter sistémico de la competitividad y la naturaleza interactiva de la innovación (Morgan, 1995) concebida como un proceso de aprendizaje en el que se introducen nuevos conocimientos o se combinan conocimientos existentes para generar nuevas competencias (Lundvall, 1992; Gregersen y Johnson, 1996), en los últimos veinte años ha venido adquiriendo nueva significación el papel del entorno local y de sus instituciones en el desarrollo de la capacidad innova- 
dora de las empresas. En este nuevo escenario internacional, ambiente local y economía global no son términos antagónicos, ya que la globalización debe su fuerza a la complejidad del conocimiento y de la sinergia que se produce a partir de la confrontación competitiva de diferentes variantes territoriales y de redes de agentes. ${ }^{20}$

Desde ese punto de vista, el ambiente local se entiende como el conjunto de instituciones y agentes locales y sus relaciones recíprocas; las características que presenta son decisivas para el desarrollo de la capacidad creadora de las empresas. Puede considerársele como un espacio público que, en su aspecto positivo, puede dar lugar a fenómenos de eficiencia colectiva, definidos como las ventajas competitivas derivadas de economías externas y de la acción conjunta de los agentes (Camagni, ed. 1991; Bianchi y Miller, 1994). ${ }^{21}$ La presencia de un entorno favorable puede traducirse en acciones de los agentes que componen la sociedad civil, los que a partir de la cooperación, la competen-

\footnotetext{
${ }^{20}$ La interconexión de los mercados financieros internacionales asociada al proceso de globalización cataliza esos procesos.

21 En Boscherini, Malet Quintar y Yoguel (1997) se define un gradiente de ambientes locales teóricos con desigual externalidad en función de las características de los agentes, la lógica del sistema y las estrategias de los agentes, así como el grado de vinculación interna y externa de todos los elementos componentes. Los ambientes en los que se generan las máximas externalidades positivas son los que presentan las mejores características en los tres planos señalados. En estos ambientes, predominan muchos agentes (elevado número de eslabones, sectores y empresas) con modalidades productivas heterogéneas y con escasa integración vertical, lo que favorece la cooperación empresarial y el desarrollo de distintas formas de externalización. Por su parte, el grado de cobertura de las instituciones (educativas, informativas y de servicios) es alto, lo que es potenciado por una adecuada complementariedad. En este tipo de ambiente teórico puede identificarse una lógica única consistente en conservar el núcleo del negocio y las estrategias empresariales tendientes a la globalización, a la descentralización y en algunos casos a la reintegración vertical de las fases críticas. Los agentes del área se autoorganizan sin coordinación visible y constituyen un espacio público avanzado. Los agentes cooperan en actividades productivas, comerciales, de servicios y en la solución de problemas. Las instituciones educativas llevan a cabo trabajos de investigación y desarrollo en conjunto con las empresas y los servicios que ofrecen están ajustados a las necesidades de los agentes, en el marco de una fuerte interacción. Por último, las relaciones con el entorno exterior del medio se basan en vínculos entre grupos de instituciones y empresas del área con similares ubicados fuera del sistema local. En el otro extremo teórico, el medio negativo genera las mayores externalidades negativas posibles para las empresas. Se caracteriza por la presencia de pocos agentes con conductas homogéneas que operan con elevada integración vertical en el marco de un sistema institucional ubicado por debajo de los requerimientos mínimos necesarios para el desarrollo de la competencia individual y colectiva. El sistema no opera ni con una lógica ni con estrategias predominantes. Sin agentes coordinadores, las empresas no se autoorganizan como en el caso anterior. Por último,
} los agentes no tienen relaciones con el exterior del sistema local. cia y la presión mutua generan una tensión colectiva que favorece el desarrollo de estrategias innovadoras y minimizan las diferencias entre agentes. La experiencia internacional sugiere que cuando estos ambientes tienen un comportamiento positivo actúan como un operador de cuasimercado que disminuye las incertidumbres dinámicas, contrarresta las debilidades de la cultura organizacional, potencia los procesos de aprendizaje, suministra las competencias faltantes a los agentes, contribuye al proceso de difusión de conocimiento codificado y tácito y tiende a disminuir la desigualdad social (Camagni, ed., 1991).

De esta manera, en los ambientes positivos se va generando un "capital social" construido a partir de vinculaciones complejas que se manifiestan en el desarrollo de la confianza recíproca entre los agentes (Morgan, 1995), lo que facilita la reducción de la incertidumbre y la difusión de conocimientos codificados y tácitos. Este tipo de ambiente es el resultante de procesos de aprendizaje individuales y colectivos. Aunque un medio positivo tiende a igualar las conductas de los agentes, su presencia no implica un beneficio automático para todos ellos. Así, para poder aprovechar las externalidades del medio las empresas deben sobrepasar un umbral mínimo de competencias y de mecanismos endógenos de generación y transmisión, sin los cuales no ocurre el proceso de aprendizaje y de transformación de conocimiento. La generación de estas competencias en los ambientes locales es también la consecuencia de un proceso evolutivo de creación y destrucción de rutinas y convenciones (Gregersen y Johnson, 1996). Por lo tanto, aprender también requiere desaprender. En ese proceso, las empresas modifican el medio con la transformación interna del conocimiento y la forma como se vinculan con otros agentes. El desarrollo de procesos de aprendizaje en ambientes locales depende de la trayectoria que sigan los agentes en su evolución, del patrón tecnológico del sector al que concurren y del grado de desarrollo del medio (Camagni, ed., 1991).

Para valorizar sus especificidades (conocimientos, capacidades), los espacios locales y nacionales deben ser cotejados a escala mundial. Rullani (en prensa) ha planteado claramente la interacción entre los rasgos codificados y tácitos del proceso de generación de conocimiento, en especial cuando se considera el papel que desempeña el ambiente. Como los elementos tácitos tienen un fuerte componente contextual y de experiencia, en el mundo globalizado de hoy la dimensión territorial tiene un lugar destacado en la generación de conocimientos. El carácter contextual, experi- 
mental y evolutivo del conocimiento lleva no sólo a la generación de conocimientos tácitos, sino también a su codificación para poder transformarlos y eventualmente transferirlos. De esta manera, el conocimiento puede circular fuera de un contexto y asumir una forma desterritorializada, pero para aplicarlo efectivamente en otro contexto debe ser recontextualizado.

Entonces, desde el punto de vista territorial hay dos polos en el circuito cognoscitivo: i) la dimensión global, que abarca el proceso de producción, transferencia y uso del conocimiento fuera de su contexto (codificado) y ii) la dimensión local, que incluye el proceso de aprendizaje y sedimentación, cuando el conocimiento se arraiga en el territorio. Así, según Rullani (en prensa), la generación del conocimiento de los agentes económicos se produce en un contexto local y específico. La salida del contexto y la transferencia del conocimiento requieren una codificación previa. En esa etapa, el conocimiento adquiere un carácter global y abstracto. Sin embargo, para que ese conocimiento abstracto sea útil debe ser recontextualizado y sometido a un proceso de adaptación que haga posible la creación de conocimiento tácito.

Este proceso de codificación y decodificación del conocimiento que vincula lo local y lo global es un producto de lo que Becattini y Rullani (1993) denominan integración versátil; ellos señalan que la eficacia de las diversas formas de integración (tecnológicas, organizacionales y comunicacionales) no sólo depende de la eficiencia de los códigos empleados por los distintos agentes sino fundamentalmente de capacidades y habilidades adquiridas por experiencia directa que no pueden expresarse en códigos estandarizados. Un aspecto que adquiere una importancia vital en el proceso de generación y circulación del conocimiento tácito en los ambientes locales es la forma de articulación del lenguaje formal e informal entre los agentes (Poma, en prensa). Mientras el conocimiento codificado se aprende en forma idéntica, el conocimiento tácito pasa por el proceso de interpretación de quien aprende y difiere por lo tanto del conocimiento del agente que lo ha transferido: esta discrepancia entre trasmitir/hacer circular y aprender le agrega algo al conocimiento, innova en él y también innova en el lenguaje (ibid.).

\section{Los procesos de aprendizaje en los sistemas de empresas}

Los aspectos examinados con relación al proceso de generación y circulación del conocimiento dentro de un ambiente determinado se reproducen en los sistemas de empresas con la especificidad derivada del predominio de relaciones insumo-producto horizontales y verticales entre los agentes que forman parte de ellas. Como al interior de estas redes o tramas productivas una cantidad importante de transacciones se producen fuera del mercado, los elementos tácitos pueden adquirir una importancia aún mayor.

Sin embargo, la intensidad que adquiera el proceso de aprendizaje en los sistemas de empresas dependerá de la jerarquía que se le asigne tanto al interior de la empresa como en cada uno de los agentes que la integran, y también del grado de autoorganización. La importancia alcanzada por los elementos tácitos desarrollados en los distintos sectores productivos depende de cuán profundos sean el intercambio de experiencias y el trabajo conjunto, de la importancia que tenga el trabajo en células, de la circulación de la información en cada uno de los componentes de la red productiva y de la interacción con el entorno local.

Una singularidad de las redes productivas es la sinergia que puede adquirir en ellas el proceso de aprendizaje, lo que puede manifestarse en la generación de una mayor cantidad y complejidad de elementos tácitos que se constituyen en barreras de entrada para los agentes que no pertenecen a la red. Así, si los agentes $h \mathrm{y} j$, cuyas ventajas competitivas fueron descritas en la sección anterior, pertenecen a la trama $T i$, la ventaja competitiva agregada de la trama será mayor a la suma de las funciones de los agentes que la integran, generándose mejoramientos de eficiencia que pueden asimilarse a las economías de escala y de variedad, consideradas en forma simultánea. Sin embargo, junto con el desarrollo conjunto de conocimientos de tipo tácito, en los sistemas de empresas se produce una codificación del pensamiento tácito para que éste pueda circular a través de lenguajes formales (Poma, en prensa). ${ }^{22}$

El desarrollo de los procesos de aprendizaje al interior de los sistemas de empresas no sólo introduce cambios en las actividades de capacitación requeridas sino además en la forma en que se formulan los contratos y en los incentivos implícitos. En las relaciones formales e informales que se desarrollan al interior de una red productiva, los contratos y la fijación de precios implícitos en los intercambios van adquiriendo mayor precisión a partir de las sinergias generadas en

\footnotetext{
22 Según Poma, esto elimina las discontinuidades características de la interpretación subjetiva del conocimiento tácito y reduce a su interior las innovaciones incrementales, produciendo procesos de ruptura que permiten el desarrollo de innovaciones radicales.
} 
el proceso de aprendizaje conjunto. Mientras las exigencias mínimas de los contratos (precisión) estarían positivamente asociadas al nivel alcanzado en el proceso de aprendizaje, la tasa de incentivos mostraría una relación inversa con el nivel de aprendizaje: cuanto mayor conocimiento se alcanza menores son los incentivos necesarios para que los contratos funcionen. La evolución de los contratos supone que los agentes no sólo aprenden sino que avanzan (hay cambios de rutinas, introducción de nuevas prácticas productivas, inversiones no recuperables, etc.); este proceso no tiene marcha atrás y determina las posiciones iniciales en los nuevos contratos En suma, mientras la tasa de riesgo tiene una relación inversa con el desarrollo de procesos de aprendizaje, la complejidad alcanzada por los contratos tiene una relación directa con él. Por lo tanto, como la tasa de incentivos necesaria para la formulación de los contratos depende del nivel de riesgo, el desarrollo de procesos de aprendizaje desempeña un papel decisivo - al interior de la red productiva - para disminuir la cantidad de incentivos necesarios para que funcione el sistema de contratos. ${ }^{23}$

\section{V}

\section{Algunas reflexiones finales}

Como se señaló en las secciones anteriores, en el nuevo escenario internacional la creación de ventajas competitivas de los agentes depende del desarrollo de procesos de aprendizaje que tienen características sistémicas. En su desarrollo influyen no sólo el conjunto de características individuales de los agentes, sino también el grado de articulación de los sistemas locales y de las tramas productivas de las que forman parte. Desde ese punto de vista, en la generación y la circulación del conocimiento codificado y tácito tanto al interior de las organizaciones como entre ellas influye poderosamente la complejidad de los nexos y el grado de cooperación tecnológica formal e informal entre los agentes; el tipo de vínculos entre las universidades, los centros de investigación y las empresas; la preparación de los recursos humanos y la complejidad del sistema educativo y de capacitación; y el grado de desarrollo de los agentes intermediarios (capital de riesgo, servicios técnicos específicos, asociaciones profesionales, grupos de ex alumnos, etc.) que actúan además como mensajeros (enlaces) en el proceso de transmisión y generación de informaciones. Como en este nuevo panorama la creación y transferencia de conocimientos se desarrolla en muchas formas, se producen áreas informales de innovación al interior de las empresas e instituciones que complementan la actividad de los laboratorios dedicados específicamente a la investigación y el desarrollo.

Estos procesos no son la consecuencia de una evolución natural y lineal de los sistemas productivos sino que requieren un largo período de maduración. Deben interpretarse como el resultado de un proceso de construcción económica y social compleja que toma tiempo y tiene una evolución histórica, sobre el que influyen múltiples planos. Además de los factores formales e informales de naturaleza tecnológica y económica, influyen otros elementos, entre los que destacan,

\footnotetext{
${ }^{23}$ El grupo de mejora continua de Volkswagen ofrece un ejemplo interesante de un sistema de asistencia técnica y capacitación de carácter informal que apunta a desarrollar competencias a partir de los conocimientos tácitos específicos de los agentes participantes, lo que permite su movilización, su codificación y su valorización por los agentes de la trama productiva. Esto genera nuevas competencias y conocimientos que permiten reiniciar desde un nivel más alto el proceso de desarrollo del conocimiento. En el proceso de capacitación y asistencia técnica que llevan a cabo operan como un intermediario (es decir, consejero tecnológico) que permite aumentar la velocidad con que se produce la difusión del conocimiento. Como parte de la idea de que el que sabe es el "otro", se genera una integración de saberes bidireccional. En ese sentido, la capacitación consiste en un direccionamiento de los conocimientos de diferente tipo y nivel que integran - a partir de un proceso sistémico- saberes técnicos de mayor nivel con los conocimientos de las bases que trabajan en forma directa en el proceso productivo. En el proceso de mejora continua, la capacitación consiste en generar una sinergia de conocimientos de distinto tipo que posibilitan el desarrollo de economías externas: así la sinergia es mayor que los conocimientos que aporta cada uno de los integrantes de la trama. El programa de consejerías tecnológicas aplicado en Argentina por la Agencia Nacional de Promoción Científica y Tecnológica (Secretaría de Ciencia y Tecnología), orientado hacia empresas pequeñas y medianas, tiene una concepción similar. Los consejeros tecnológicos de grupos de empresas parten de las competencias de los agentes e intervienen conjuntamente con éstos en el desarrollo de su gestión tecnológica. Se parte de la idea de que debido a las fallas existentes en el mercado de tecnología, la demanda de las empresas no está dirigida tanto a una consultoría técnica particular sino a identificar las cuestiones claves para el desarrollo de las competencias y de la capacidad de innovación de las empresas (véase Argentina, Presidencia de la Nación, Secretaría de Ciencia y Tecnología,
} 1997). 
el desarrollo de confianza recíproca entre los agentes, la valorización social del papel del empresario y el grado de cumplimiento de los contratos. Se trata de tendencias que, siendo aún embrionarias, tienden a imponerse en los países desarrollados y también, con mayor rezago, en los países de menor desarrollo.

En los países en desarrollo, para que el aprendizaje tecnológico sea exitoso es necesario adquirir los elementos codificados de la tecnología y desarrollar los elementos tácitos complementarios. Para cumplir con la primera condición se tropieza con fallas en el mercado de tecnología y para alcanzar la segunda se precisa una acumulación de competencias durante un proceso de evolución previo, muchas veces inexistente o trunco. En consecuencia, la generación de ventajas competitivas vinculadas a la aplicación intensiva del conocimiento y a un perfil más complejo de estructuras productivas de los países en desarrollo constituye un desafío que desborda la gestión de los agentes económicos individuales y exige medidas que involucren al conjunto de las instituciones publicas y privadas y a los demás agentes sociales.

Para alcanzar los objetivos señalados son necesarios, entre otras cosas, i) la revalorización social de los procesos de aprendizaje y educación; ii) la creación de agentes intermediarios, que actúen como transmisorestraductores entre las diversas partes del sistema, que catalicen los procesos de aprendizaje de los agentes y que contribuyan a la creacion del mercado; iii) el desarrollo de medios locales generadores de economías externas; iv)la evolución desde las ventajas competitivas individuales a las ventajas competitivas de sistemas de empresas y sistemas locales, y iv) el establecimiento de un vínculo eficaz entre la universidad y la empresa que potencie los procesos de aprendizaje codificado y tácito.

El perfil de especialización productiva de la mayoría de los países latinoamericanos se inclina por la elaboración de recursos naturales o las actividades de maquila, y, por consiguiente, casi no incluye los procesos de aprendizaje como elemento primordial para obtener ventajas competitivas dinámicas. Pero con la profundización ulterior del modelo de desarrollo, incluso sustentado en la explotación de recursos naturales, habría que prestar una mayor atención a las cuestiones aquí planteadas. Objetivos de política como el fortalecimiento de las cadenas de valor agregado, el desarrollo de la capacidad productiva para la internacionalización, la integración de las pequeñas y medianas empresas en las redes y sistemas de empresas y el logro de un mayor equilibrio territorial (Kosacoff, ed., 1997) parecen directamente ligados al desarrollo de las competencias complejas de los agentes, difíciles de obtener sin un proceso de aprendizaje importante que involucre cuestiones no sólo codificadas sino también tácitas.

\section{Bibliografía}

Acs, Z. y D. Audrescht (1988): Innovation in large and small firms: An empirical analysis, The American Economic Review, vol. 78, Nashville, Tennessee, American Economic Association, septiembre.

Argentina, Presidencia de la Nación, Secretaría de Ciencia y Tecnología (1997): Plan Nacional Plurianual de Ciencia y Tecnología 1998-2000, Documento, $N^{\circ} 1$, Buenos Aires, Programa de Capacitación Tecnológica de Pymes Industriales.

Arrow, K. (1962): The economic implications of learning by doing, The Review of Economics Studies, vol. XXIX(3), Nº 80, Londres, London School of Economics.

Becattini, G.y E. Rullani (1993): Sistema locale e mercato globale, Economia e politica industriale, No.80, Milán, Italia, Franco Angeli.

Bell, M. y K. Pavitt (1995): The development of technological capabilities, en I. ul Haque (ed.), Trade, Technology and International Competitiveness, Washington, D.C., Banco Mundial, Instituto de Desarrollo Económico (IDE).

Bessant, J. (1991): Managing Advanced Manufacturing Technology, Manchester, U.K., NCC Blackwell.

Bianchi, P. y L. Miller (1994): Innovation, collective action and endogenous growth: An essay on institutions and structural change, en F. Boscherini y L. Poma (eds.), Territorio, cono- cimiento y competitividad en el espacio global, Buenos Aires, Universidad Nacional de General Sarmiento.

Boscherini, F., M. López y G. Yoguel (1997): Sistemas locales de innovación y el desarrollo de la capacidad innovativa de las firmas: un instrumento de captación aplicado al caso de Rafaela, Documento de trabajo, No.10, Buenos Aires, Comisión Económica para América Latina y el Caribe (CEPAL)/ Universidad Nacional de General Sarmiento, Instituto de Industrias.

Boscherini, F., N. Malet Quintar y G. Yoguel (1997): Consideraciones acerca del ambiente y el desarrollo de las capacidades innovativas de las firmas, Segundas Jornadas Nacionales de la Red Pymes, La Plata, Argentina, Centro de Estudios Bonaerense (CEB).

Boscherini, F. y G. Yoguel (1996a): La capacidad innovativa y el fortalecimiento de la competitividad de las firmas: el caso de las PYMEs exportadoras argentinas, Documento de trabajo, $\mathrm{N}^{\circ} 71$, Buenos Aires, Oficina de la CEPAL en Buenos Aires. (1996b): Algunas consideraciones sobre la medición de los procesos innovativos: la relevancia de los rasgos informales e incrementales, Redes, Buenos Aires, Universidad Nacional de Quilmes, Centro de Estudios e Investigaciones.

Camagni, R., ed. (1991): Innovation Networks: Spatial Perspectives, Londres, Belhaven Press. 
Cariola, M. y A. Quiroz (1998): Competencias generales, competencias laborales y curriculum, Montevideo, Centro Interamericano de Investigación y Documentación sobre Formación Profesional (CINTERFOR), Organización Internacional del Trabajo (OIT).

CEPAL (Comisión Económica para América Latina y el Caribe) (1996): Quince años de desempeño económico. América Latina y el Caribe 1980-1995, Santiago de Chile.

Cimoli, M. y G. Dosi (1994a): De los paradigmas tecnológicos a los sistemas nacionales de producción e innovación, Comercio exterior, vol. 44, $\mathrm{N}^{\circ} 8$, México, D.F., Banco Nacional de Comercio Exterior (BANCOMEXT).

(1994b): Technological Paradigms, Patterns of Learning and Development: an Introductory Roadmap, Londres.

Dal Bó, E. y B. Kosacoff (1998): Líneas conceptuales ante evidencias microeconómicas de cambio estructural, en B. Kosacoff (ed.), Estrategias empresariales en tiempos de cambio: el desempeño industrial frente a nuevas incertidumbres, Buenos Aires, Universidad Nacional de Quilmes/Oficina de la CEPAL en Buenos Aires.

David, P.A. (1985): CLIO and the economics of QWERTY, The American Economic Review, vol. 75, Nㅜㄹ, Nashville, Tennessee, American Economic Association.

Ducatel, K. (1998): Learning and skills in the knowledge economy, Documento de trabajo, $\mathrm{N}^{\circ} 2$, Aolborg, Dinamarca, Danish Research Unit for Industrial Dynamics (DRUID).

Ernst, D. y A. Lundvall (1997): Information technology in the learning economy, challenges for developing countries, Documento de trabajo, $\mathrm{N}^{\circ} 12$, Aolborg, Dinamarca, DRUID.

Gallart, S. (1998): Competitividad, redes productivas y competencias laborales, Montevideo, CINTERFOR, OIT.

Gómez, R. (1995): Neoliberalismo y seudociencia, Buenos Aires, Editorial Lugar.

Gregersen, B.y B. Johnson (1996): Learning economies, innovation systems and European integration, Regional Studies, vol. 31, Oxford, Reino Unido, Pergamon Press .

Grossman, G. M. y E. Helpman (1992): Innovation and Growth in the Global Economy, Cambridge, Massachusetts, MIT Press.

Gutman, G. (1999): El sector agropecuario y el sistema agroalimentario: nuevos procesos, Buenos Aires, Instituto Torcuato di Tella, Centro de Estudios Urbanos y Regionales (CEUR).

Hanser, L. (1995): Traditional and Cognitive Job Analyses as Tool for Understanding the Skill Gap, Berkeley, California, Universidad de California, National Center for Research in Vocational Education.

Johnson, B. y B.A. Lundvall (1994): Sistemas nacionales de innovación y aprendizaje institucional, Comercio exterior, vol. 44, $\mathrm{N}^{\circ}$ 8, México, D.F., BANCOMEXT.

Kosacoff, B., ed. (1997): Estrategia de desarrollo empresarial, Buenos Aires, Oficina de la CEPAL en Buenos Aires.

Lakatos, I. (1983): La metodología de los programas de investigación científica, Madrid, Alianza Editorial.

Lall, S. (1992): Technological capabilities and industrialization, World Development, vol. 20, $\mathrm{N}^{\circ}$ 2, Oxford, Reino Unido, Pergamon Press.

(1995): The creation of comparative advantage: the rol of industrial policy, en I. ul Haque (ed.), Trade, Technology and International Competitiveness, Washington, D.C., Banco Mundial, IDE.

Lam, A.(1998): Tacit knowledge, organizational learning and innovation: A societal perspective, Documento de trabajo, $\mathrm{N}^{\circ}$ 22, Aolborg, Dinamarca, DRUID.

Lassini, A. (1992): Il ruolo dell'attività di R\&S per lo sviluppo della competitività delle PMI, en F. Onida, G. Viesti y A.M. Falzoni (eds.), I distretti industriali: crisi o evoluzione?, Milán, Egea.

Lundvall, B.A. (1992): The learning economy: Challenges to economic theory and policy, trabajo presentado a EAEPE Conference, Copenhague, European Association for Evolutionary Political Economy (EAEPE).
Malerba, F. (1993): National system of innovation: The case of Italy, en R. Nelson (ed.), National Innovation System, Oxford, Reino Unido, Oxford University Press.

Malerba, F. y L. Orsenigo (1993): L'accumulazione delle capacita'tecnologiche nell'industria italiana (1969-1984), en L. Filippini (ed.), Innovazione tecnologica e servizi alle imprese, Milán, Italia, Franco Angeli.

Mertens, L. (1996): Competencia laboral: sistemas, surgimiento y modelos, Montevideo, CINTERFOR, OIT.

Metcalfe, S. (1998): Evolutionary Economics and Creative Destruction, Manchester, Routledge.

Moori-Koenig, V. y G. Yoguel (1998): Capacidades innovadoras en un medio de escaso desarrollo del sistema local de innovación, Comercio exterior, vol. 48, N 8, México, D.F., BANCOMEXT.

Morgan, K. (1995): The Learning Region: Institutions, Innovation and Regional Renewal, Cardiff, University of Wales.

Nelson, R. (1991): Why do firms differ and how does it matter?, Documento de trabajo, $\mathrm{N}^{\circ} 7$, Berkeley, California, Universidad de California, Center in Research Management.

Nightingale, P. (1996): A cognitive model of innovation electronic, Documento de trabajo, $\mathrm{N}^{\circ} 11$, Sussex, Science Policy Research (SPRU).

Nonaka, I. y H. Takeuchi (1995): The Knowledge-Creating Company: How Japanese Companies Create the Dynamics of Innovation, Londres, Oxford University Press.

Novick, M. (1998): Estrategias y capacitación y aprendizaje de las firmas. Reflexión sobre empresas innovadoras en Argentina Brasil y México, trabajo presentado al Proyecto Conjunto CEPAL-GTZ sobre Políticas para mejorar la calidad, eficiencia y relevancia del entrenamiento profesional en América Latina y el Caribe, Santiago de Chile, CEPAL.

Novick, M., M. Bartolomé, M. Buceta, M. Miravalles y C. Senén González (1998): Nuevos puestos de trabajo y competencias laborales, Papeles de la Oficina Técnica, $\mathrm{N}^{\circ}$ 6, Montevideo, CINTERFOR, OIT

Novick, M. y L. Gallart (1998): Competitividad, redes productivas y competencias laborales, Ginebra, OIT.

Pérez, C. (1983): Structural change and the assimilation of the new technology in the economic and social system, Futures, vol. $15, \mathrm{~N}^{\circ}$ 5, Surrey, Reino Unido, IPC Science and Technology Press.

(1985): Microelectronics, long waves and world structural change: New perspectives for developing countries, World Development, vol. 13, $\mathrm{N}^{\circ} 3$, Oxford, Reino Unido, Pergamon Press.

Poma, L. (1998): La nuova competizione territoriale, Politica e organizzazione, Quaderni aroc, $\mathrm{N}^{\circ} 1$, Bolonia, Italia, Il Mulino.

(en prensa): La producción de conocimiento. Nuevas dinámicas competitivas para el territorio, conocimiento y competitividad en el espacio global, en F. Boscherini y L. Poma (eds.), Territorio, conocimiento y competitividad en el espacio global, Buenos Aires, Universidad Nacional de General Sarmiento.

Rearte, A., E. Lanari y P. Alegre (1997): Sistemas de innovación y el desarrollo de la capacidad innovativa de las firmas: el caso de Mar del Plata, Buenos Aires, Universidad Nacional de Mar del Plata.

Rullani, E. (en prensa): El valor del conocimiento, en F. Boscherini y L. Poma (eds.), Territorio, conocimiento y competitividad en el espacio global, Buenos Aires, Universidad Nacional de General Sarmiento.

Samuelson, P. (1962): Parable and realism in capital theory: The subrogate production function, The Review of Economics Studies, vol. XXIX(3), N 80, Londres, London School of Economics.

Solow, R. (1963): Capital Theory and the Rate of Return, Amsterdam, Países Bajos, North-Holland Publishing Company. 